\title{
Análisis de la existencia de una cultura de la escucha organizacional, desde los directivos en el sector multitiendas en Chile
}

\author{
Analysis of the existence of a culture of organizational listening, \\ from managers in the multi-store sector in Chile
}

\author{
Claro, C. ${ }^{1}$ \\ Recibido: 21-09-2020 - Aceptado: 5-02-2021 \\ https://doi.org/10.26441/RC20.1-2021-A4
}

RESUMEN: La comunicación en las organizaciones se torna cada día más compleja dado que la realidad de las empresas adquiere una fisonomía, que exige de ellas una mayor responsabilidad, al mismo tiempo que una gestión más eficiente y comprometida con los públicos con los que interactúa y una relación con ellos que tiene un carácter más permanente.

Es en este entendido que la escucha organizacional se torna como un intangible necesario de trabajar en ellas, ya que se requiere entender de mejor modo y de forma más directa cuáles son los requerimientos de los públicos con los que interactúan, generar vínculos y adaptarse, lo que se constituye como una dimensión importante de la gestión de las compañías.

El propósito de este estudio es conocer las percepciones de ejecutivos acerca de si se está realizando procesos de escucha organizacional en el sector retail en Chile y si se ha adoptado una cultura que propicie la ejecución de ella. Con este fin se entrevista directivos que trabajan en las áreas de marketing, comunicaciones y dirección de personas, se realizan cuestionarios y análisis de documentos oficiales con el fin de conocer el estado de la cuestión y si la escucha efectiva tiene consecuencias positivas para las organizaciones.

Palabras clave: comercio minorista; escucha organizacional; comunicación estratégica; cultura de escucha.

ABSTRACT: Communication in organizations is becoming more complex every day since the reality of companies acquires a physiognomy, which demands greater responsibility from them, at the same time as a more efficient and committed management with the public with whom it interacts and a relationship with them it has a more permanent character.

It is in this understanding that organizational listening becomes a necessary intangible to work on them, since it is necessary to understand in a better and more direct way what are the requirements of the public with whom they interact and generate links, what is it constitutes an important dimension of company management.

The purpose of this study is to know the perceptions about whether organizational listening processes are being carried out in the retail sector in Chile and whether a culture that encourages its execution has been adopted. For this purpose, executives working in the areas of marketing, communications and people management are interviewed, questionnaires are carried out and official documents are analyzed in order to know the state of the matter in order to know the state of the matter and if effective listening has positive consequences for organizations.

Keywords: retail; organizational listening; strategic communication; listening culture.

\footnotetext{
${ }^{1}$ Cecilia Claro es Doctora en Comunicación, Master in Business and Administration y Periodista por la Universidad de Los Andes (Chile). Es profesora de las asignaturas relacionadas con la Economía y Empresa en la Facultad de Comunicación de la Universidad de los Andes. cclaro@uandes.cl, https://orcid.org/0000-0002-3428-0616
} 


\section{Introducción}

Las empresas se desenvuelven en un contexto en el cual han adquirido un rol relevante en la sociedad no sólo desde el punto de vista de su actividad comercial, sino también desde el aporte social y cultural que realizan. El gobierno corporativo no sólo debe preocuparse de la producción, sino de otros ámbitos como son el desarrollo humano, las relaciones interculturales, la responsabilidad social y ambiental, y la creación de confianza desde y hacia la empresa (Gutiérrez-García, 2010). Las compañías procuran diferenciarse, por aspectos relativos a su talento profesional, su cultura e identidad; los modos de ser y hacer, que inspiran las políticas y decisiones y que ayudan a las organizaciones a proyectarse socialmente e inmiscuirse con la comunidad en la que interactúan.

Es en este entorno en el que para las empresas no basta sólo con vender productos, y en el que la comunicación y el desarrollo de ciertos intangibles por parte de las compañías cobra un rol relevante. La gestión relacional que despliegan las organizaciones supone para ellas trabajar en mayor medida conceptos como la reputación, la imagen, la transparencia, la confianza, la responsabilidad y aquello que es objeto de estudio de esta investigación: la escucha organizacional. Es fundamental que las compañías se planteen, revisen y reformulen de forma constante aspectos como la identidad, cómo gestionan y presentan su marca, cuál es su responsabilidad con sus públicos y cuál es su aceptación social (Villagra; López \& Monfort, 2015). Esto requiere desarrollar una escucha organizacional que comprende una cultura, políticas, estructuras, procesos, fuentes, habilidades, tecnologías y prácticas a ser implementadas en la organización (Macnamara, 2016).

A partir de lo anterior es que el objetivo de esta investigación es analizar cómo se percibe que se está realizando la escucha organizacional, desde la visión de los directivos a partir de la experiencia de cinco empresas que operan multitiendas en Chile. La selección de esta industria no es casual, sino que responde a la necesidad que ella tiene de relacionarse estrechamente con sus diferentes públicos y de manera especial con sus clientes y colaboradores. Son los consumidores quienes compran de forma online y offline las 24 horas del día y desde cualquier punto del planeta y por lo tanto quienes afectan directamente la supervivencia de las compañías de venta al detalle. Al mismo tiempo quienes trabajan en ella son factores fundamentales para entender los procesos de compra y las demandas e intereses del público al que se atiende.

En los siguientes apartados se ofrece en primer lugar un marco teórico que procura construir una noción sobre la escucha organizacional desde la disciplina de la comunicación bajo la perspectiva de diferentes autores. A continuación se contextualiza sobre qué es el concepto de cultura de la escucha en las organizaciones y los elementos que la conforman.

En segundo lugar se presenta la metodología de investigación utilizada la que es de carácter mixta y que incluye herramientas de análisis cuantitativo y cualitativo. Para ello, se realizaron entrevistas semiestructuradas a ejecutivos que se desempeñan en las cinco empresas seleccionadas, de modo de conocer sus percepciones sobre cómo se realiza la escucha; en segundo lugar se tomaron cuestionarios para levantar información concreta de las acciones para escuchar que se ejecutan en las organizaciones de forma permanente y en tercer lugar se realizó un análisis de los contenidos relacionados con la escucha que publican las empresas en sus documentos oficiales.

\section{Marco Referencial. Hacia un concepto de escucha organizacional}

Una de las misiones más relevantes de la Comunicación Estratégica en una institución o empresa, es la de producir adhesión de parte de su público objetivo, y su entorno que es relevante para su acción (Burgos, 2018). En la comunicación de las organizaciones, el trabajo por la gestión de intangibles entre los que se encuentra la escucha organizacional se fundamenta en relación a la eficiencia y 
eficacia de estrategias, la multiplicidad y multidireccionalidad de medios utilizados y la interacción con stakeholders (Apolo et al, 2017).

Es posible señalar que el énfasis de la escucha, en términos organizacionales se ha puesto sobre todo en los conceptos de voz y habla, más que en oír (Macnamara, 2014). Es así que escuchar al interior de las organizaciones justifica un análisis crítico (Macnamara, 2019). Esto lleva a procurar entender la escucha desde su relación con el diálogo, ya que el diálogo ayudaría en variadas ocasiones a equilibrar la relación con los públicos colaborando en la co-creación de significados que nutren la construcción social (Botan \& Taylor, 2004). El diálogo, como describen Grunig y Hunt (1984), está orientado, en última instancia, a la comprensión mutua y el entendimiento entre la organización y sus públicos, ya que las instituciones y los públicos pueden persuadirse mutuamente para modificar sus actitudes y conductas. La adecuada toma de decisiones hecha por las entidades será mejor cuando están abiertas a feedback (Taylor \& Kent, 2014), ya que esto genera eventualmente en las personas confianza y seguridad para expresar sus ideas, aunque las resoluciones que se tomen no siempre serán las más convenientes bajo la mirada de la directiva de las organizaciones.

El diálogo visto desde la perspectiva estratégica y del management de la organización apunta a la relación con los diferentes grupos de interés, ya que la vinculación tiene un beneficio mutuo para ambas partes, genera confianza en la percepción de los stakeholders y favorece la actividad de la compañía (Gutiérrez-García, Recalde \& Piñera-Camacho, 2015). El diálogo se refiere a procesos de comunicación bidireccionales en los que se abordan intereses y preocupaciones en conflicto (Susan-

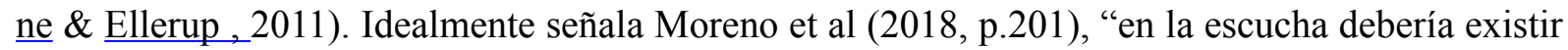
siempre un enfoque dialógico, ya que el diálogo se produce cuando la escucha y el entendimiento se dan a la vez". La escucha activa hace que el interlocutor se sienta escuchado, y permite crear empatía, poniéndonos en su lugar y siendo comprensivo (Romero-Rodríguez \& Castillo Abdul, 2019). La escucha, desde esta mirada, establece el proceso dialogal como el "modelo básico de cualquier consenso", en el que, si hay algún indicio de superioridad, se plantearía una incoherencia (Rojas, 2017, p 192)

Por otra parte, si se considera el diálogo desde el punto de vista de la teoría retórica trabajada por (Heath et al., 2006), habría dos factores que lo construyen y que son relevantes para los stakeholders: acordar qué constituye diálogo y qué lo diferencia de otras formas de comunicación y por otra parte transparentar los motivos que hay tras el diálogo emprendido.

Entendida la escucha desde un proceso dialógico, ésta presenta diferentes aproximaciones conceptuales. Desde el ámbito de las compañías, Borner \& Zerfas (2018), se refieren a ella desde la óptica de la estrategia de la comunicación de la empresa y que consta de varias estructuras, estrategias, procesos, y actividades que se integran en el flujo y gestión de las comunicaciones corporativas. Una organización que escucha es la que se preocupa de desarrollar y mantener una relación simétrica de dos direcciones con sus públicos (Worthington \& Fitch-Hauser, 2018).

Bussey (2010) plantea una definición de la escucha a partir del diálogo y sugiere tratar a las personas como objetivos y no como fines. Flynn, Valikoski \& Grau (2008) se acercan a una definición desde la empresa y la imagen y la explican como una combinación de las habilidades de escucha de un empleado y el entorno en el que se produce la escucha, la que es moldeada por la organización y es, por tanto, una de las características de la imagen de la organización. Claro (2019) la plantea como una acción y proceso continuo que se realiza a través de diferentes instrumentos formales e informales y orienta a conocer cuáles son los intereses y demandas de los públicos.

El objeto de la escucha entonces buscaría mejorar la comprensión mutua entre las personas y en el caso de las organizaciones ayudaría a una adecuada toma de decisiones y a generar un mayor vínculo con los trabajadores y consumidores. Si se asume que la sociedad demanda de la empresa algo 
más que la venta de bienes, la relación entre individuos y entidades adquiere un valor crucial y los gobiernos corporativos deben buscar la satisfacción de las necesidades de ambos. Por lo anterior, la comunicación interpersonal es fundamental en la formación de relaciones, ya que las personas al compartir los roles de emisor y receptor se conectan para crear significado (Trenholm, \& Jensen, 2008).

Con el fin de detallar lo que implica una escucha en las instituciones es que Macnamara incorpora en su definición una estructura que ha denominado Arquitectura de la Escucha y que comprende ciertos elementos que tienen que estar presentes en la organización:

La cultura, las políticas, la estructura, el proceso, los recursos, las habilidades, las tecnologías y las prácticas empleadas por una organización para dar reconocimiento, atención, interpretación, comprensión, consideración y respuesta a sus grupos de interés y públicos. (Macnamara, 2016, p.52).

La cultura será explicada en párrafos siguientes, pero vale detenerse en algunos conceptos de esta definición que plantea Macnamara (2016). En el caso de las políticas para escuchar se refiere a directivas y lineamientos sobre cómo la escucha debe ser conducida; las políticas de escucha relacionadas con el reconocimiento y establecer a quién se escucha; la estructura y procesos apunta a cómo se efectúa y quien es el responsable de ella; las tecnologías para escuchar pueden colaborar a realizar mejor escucha, pero no son el único elemento que facilita la ejecución de ella. Los recursos en tiempo, personas y presupuestarios son importantes para poder efectuarla.

Para entender mejor el concepto de escucha y determinadas las principales características de ella nos enfocaremos en la noción de la cultura organizacional que apunta a escuchar. Borner \& Zerfass (2018) señalan que la cultura en la organización implica estructuras de orientación colectiva que influyen significativamente en el comportamiento de los empleados. Si la escucha forma parte de la cultura corporativa, todos los empleados debieran estar sensibilizados para escuchar las necesidades e impulsos de las partes interesadas con quienes están en contacto.

Al mismo tiempo, una cultura organizacional implica un patrón de consideraciones básicas, conocidas por un grupo para resolver sus problemas tanto externos como internos, resultado de un proceso de grupo parcialmente influenciado por un líder y que supone una organización en cambio permanente, orientada al diálogo (Schein 2010, 1993 y 1992).

Una cultura organizacional ayuda a entender a la institución (Purdy \& Manning, 2015), genera conocimiento al interior de ella (Strandberg, 2010), mejora los índices de confianza, (Gutiérrez \& Recalde, 2010) y contribuye a la construcción de relaciones entre los diferentes públicos (Journee \&Weber, 2017). Gillchrist \& Van Hoeven (2012) señalan que la escucha puede ser una característica de la cultura organizacional y un valor al interior de la organización que se comanda, se decide por alguien y que trata de medirse. Al mismo tiempo, cuando se crean y mantienen culturas en la organización que facilitan la escucha se empodera a los empleados y mejora el desempeño (Brownell, 2008).

Comprender qué significa comunicar y qué es lo que comprende influiría en las interacciones de la organización y en concreto en los procesos relacionados con la escucha. Por lo anterior es relevante que los directivos de la organización y el equipo de comunicaciones reconozcan que diferentes grupos e individuos de ella tienen derecho a hablar y pueden ser comprendidos y considerados (Macnamara, 2016 y 2015). Así para que una institución pueda generar el vínculo con los públicos que tienen relación con ella, el compromiso y lograr una lealtad con la marca, importaría desarrollar una cultura de escucha enfocada en reconocer a otros, prestar atención, permitir hablar, tratar de entender lo que manifiestan y comprender en la medida de lo posible lo que los públicos están transmitiendo. Se busca así, una relación que capture confianza, compromiso y satisfacción de modo de crear sinergias positivas (Palmatiere et al., 2006). Lo anterior genera que las relaciones de 
marketing busquen procesos dinámicos perdurables y que permitan alcanzar a diferentes públicos al interior y exterior de la organización (Palmatiere, 2008).

En el caso de retail que apunta al consumo Itani, Goad, \& Jaramillo (2019) señalan que: "Una perspectiva relacional del proceso de decisión de compra requiere una comunicación diádica basada en una escucha efectiva". Asimismo, la escucha del vendedor al cliente genera una satisfacción y confianza por parte del consumidor (Janakiraman et al, 2019).

\section{Metodología}

\subsection{Diseño de la Investigación}

Como objetivo general de esta investigación se plantea analizar -desde la óptica de un grupo de ejecutivos de las empresas del sector multitiendas de retail chilenos- cual es su percepción sobre la presencia de una cultura de la escucha en las organizaciones en las que trabajan. La pregunta de investigación apunta a conocer si las organizaciones de retail chileno consideran la escucha como un elemento vertebral de su gestión y que forma parte constitutiva de la cultura organizacional definida por ellas.

Los grupos de interés que se pueden estudiar para esta industria son clientes o consumidores; colaboradores o trabajadores, accionistas o propietarios, proveedores, medios de comunicación, autoridades locales o nacionales y comunidades con los que se relacionan en las distintas localidades donde se ubican. Se consideró para esta investigación acotarlo solo a dos grupos de interés con los que el retail se relaciona: consumidores y colaboradores. Para esto se tuvo en consideración cuáles eran los grupos que de forma transversal afectaban a todas las tiendas por departamento estudiadas sin importar el tipo de propiedad, tamaño u origen.

Con el objeto de circunscribir el análisis, se escogió una metodología de carácter mixto -que incluye instrumentos cualitativos y cuantitativos- y que se concentra preferentemente en entrevistas semiestructuradas con un guion de nueve preguntas que buscan obtener información de las impresiones de los ejecutivos acerca de cómo están escuchando a consumidores y colaboradores. La muestra se acotó e incluyó a entrevistados que trabajaran en las áreas de comunicaciones, marketing y dirección de personas de cada una de las compañías. Se seleccionan estas tres áreas pues tienen una relación permanente con colaboradores y clientes en esta industria.

Para la elaboración de este trabajo se tomó como principio la metodología utilizada en "The Organizational Listening Project" realizado por Jim Macnamara (2015), estudio transdisciplinario que explora cómo las organizaciones escuchan a través de una variedad de prácticas que incluyen la audiencia, las partes interesadas y la investigación social; relaciones con los clientes; redes sociales; y consulta pública; así como relaciones públicas y comunicación empresarial, gubernamental, política, organizativa y de marketing. El estudio realizado por Macnamara fue trabajado metodológicamente a través de etnografía; entrevistas en profundidad a altos directivos en funciones clave que potencialmente escuchan a las partes interesadas; análisis de contenido de documentos relevantes de las organizaciones y en algunos casos a través de pruebas de campo para monitorear respuestas que entregaban las organizaciones a sus públicos frente a determinadas consultas.

Esta investigación se ajustó a la realidad local y de acuerdo con los objetivos y tiempos destinados para ella. Por lo anterior se utilizó como instrumentos el guión general de las entrevistas en profundidad, cuestionarios y análisis de documentos. La realización de etnografía y pruebas de campo no se consideraron para esta etapa de análisis por factores de tiempo y de recursos.

La selección de entrevistados realizada para este trabajo fue intencional y se compone de expertos en sus respectivas áreas de trabajo (comunicaciones, marketing y dirección de personas) lo que 
facilita un conocimiento especializado y preciso. Los entrevistados solicitaron confidencialidad de sus nombres, debido principalmente a la alta competencia de esta industria.

Del total de 43 ejecutivos que conforman el objeto de estudio se logró comunicación con $65,9 \%$ a través de contacto vía linkedin y/o e-mail; un $18 \%$ señaló no tener interés en participar de la investigación o nunca concretó la reunión, pese a insistir en ella en hasta tres oportunidades. Se entrevistó a 22 directivos en el período enero-julio de 2019 , que equivale al $54 \%$ del total.

Los cargos de los entrevistados de estas tres áreas no tienen el mismo nombre en todas las empresas, pero son homólogos para efectos del trabajo que realizan. Cabe consignar que a través de la voz del entrevistado se conocen experiencias que se constituyen como datos para esta investigación. Se busca conocer la perspectiva del sujeto estudiado, comprender cuáles son las categorías que utiliza para encuadrar el concepto de escucha, sus percepciones e interpretaciones, sin partir de ideas pre definidas (Corbetta, 2007), así como sus puntos de vista (Bryman, 2012). Las categorías utilizadas procuran responder cuál es el concepto que tienen sobre información, difusión, comunicación, escucha, bidireccionalidad.

Las entrevistas se realizaron presencialmente en las oficinas de los ejecutivos o en cafés cercanos a ellas, en el caso que no contaran con espacios privados para efectuarlas. Ellas fueron grabadas, se firmó un consentimiento informado, asegurando la confidencialidad del tema. Estas tuvieron una duración promedio que osciló entre los 50 y 75 minutos.

Como complemento a las entrevistas se tomó un cuestionario de 17 preguntas con el fin de delimitar las acciones concretas que se efectúan para escuchar y conocer los instrumentos que se utilizan en cada empresa para dichos fines. Se usó el método de preguntas categorizadas con opciones de respuesta que han sido previamente delimitadas, las que incluyen hasta cinco alternativas. Estos cuestionarios se tomaron en los 10 minutos iniciales a cada entrevista realizada a los ejecutivos. Durante el transcurso del piloto se procuró que los cuestionarios fueran respondidos con anterioridad a la misma, pero el procedimiento no funcionó, ya que los ejecutivos argumentaron falta de tiempo para responderlos, por lo que se decidió realizarlo en el tiempo otorgado a la entrevista semi estructurada.

Adicionalmente para mayor información se revisaron algunos documentos oficiales de la compañía: memorias anuales 2017 y 2018 publicadas por todas las empresas e informes de sostenibilidad en el caso que los tuvieran.

La utilización de estos tres métodos permitió la integración, con el fin de obtener una fotografía más completa del fenómeno que se trata de describir y comprender en profundidad el fenómeno en cuestión. (Hernández Sampieri, Fernández Collado \& Baptista Lucio, 2010; Denzin \& Lincoln, 1994).

El método de estrategia de análisis se efectuó del siguiente modo. En el caso de las entrevistas semi estructuradas, se transcribieron y las respuestas se agruparon de acuerdo con ocho ejes temáticos relacionados con el concepto de escucha y cultura de escucha definidos previamente. Estos ocho ejes temáticos son: si la organización considera a la escucha como un tema importante a desarrollar como organización; si la escucha es un elemento que es parte de la cultura de la organización; si la cultura de la escucha de la organización depende fuertemente de los liderazgos individuales; cuáles son los elementos a considerar para que la escucha sea efectiva; cuáles son las consecuencias positivas de efectuar la escucha en la organización; cuáles son las consecuencias negativas de efectuar la escucha en la institución; si las actividades de comunicación de la entidad fomentan las relaciones con los diferentes públicos que interactúan con ella y si hay en su entidad estructuras definidas que permiten escuchar a los diferentes stakeholders. A cada párrafo de la entrevista transcrita se la asoció con un número de 1 a 8 de acuerdo a su correspondencia con los ocho ejes temáticos enunciados. 
Las respuestas se ingresaron y trabajaron con el software Dedoose con una codificación de 8 palabras y 29 sub códigos de modo de conocer si estos temas están presentes bajo la perspectiva de los ejecutivos de la organización.

En el caso de los cuestionarios se agruparon de acuerdo con: cada respuesta si (positiva) se la identificó con el número 1, mientras que las respuestas no (negativa) fueron identificadas con un 0 . Con estos datos se hizo el cruce de información para obtener respuestas generales. En primer lugar, una caracterización que determinó empresa, cargo, sexo, profesión, edad, años en el puesto de trabajo, número de personas a cargo. Luego se procesaron los datos relativos al resto de las preguntas obteniendo datos más particulares.

En el caso de los documentos oficiales de las compañías también se ingresaron los datos al software Dedoose y los 14 documentos fueron analizados con la planilla de códigos establecida para las entrevistas semi estructuradas que se detalló anteriormente.

\subsection{Multitiendas en Chile}

Para situar el contexto, en el caso chileno el comercio general representa el 50,2\% del PIB (US\$300.000 millones en 2019), sin diferenciar en específico a qué rubro corresponde (Banco Mundial, 2020). El número de tiendas por departamento que están presentes a nivel nacional incorpora a las que venden vestuario y electrodomésticos. Se suman a ellas otras que sólo comercializan vestuario y otras que sólo están presentes es determinadas regiones del país. Con esto el número de marcas de tiendas por departamento en Chile son 12 -a agosto de 2019- algunas sociedades anónimas abiertas y otras cerradas que no se transan en bolsa.

El diseño de este estudio apunta a un análisis acotado al interior de la industria del retail chileno -en concreto multitiendas- y con un corte preferentemente cualitativo de modo de poder comprender las impresiones de ejecutivos que se desempeñan áreas relacionadas con la escucha organizacional. Las empresas estudiadas son cinco:Falabella, Ripley, París, ABC Din y La Polar. Las tres primeras -Falabella, Ripley y París- son las instituciones mayores en Chile superando cada una más del 20\% de participación de mercado, mientras las otras dos están en un segundo rango de ventas que bordea el 7\% cada una (datos año 2019).

Cabe consignar que en esta selección se considera que la propiedad está principalmente en manos de capitales chilenos. Al mismo tiempo se considera que el dominio en cuatro de ellas se concentra en familias controladoras lo que constituye estilos de administración particulares.

Otro elemento relevante y que caracteriza a todas estas compañías es que están presentes con locales comerciales a lo largo de Chile, a lo que se suma Falabella, París y Ripley que también participan en otros países de América Latina.

Por otra parte, las empresas estudiadas datan de un origen de un siglo o pocos menos de él. Esta antiguedad obliga a las entidades a adaptarse a la permanente transformación que mueva a esta industria con el objetivo de ser instituciones sostenibles y competitivas en el tiempo.

\section{Hallazgos}

Con el fin de presentar los resultados y dar respuesta a la pregunta de investigación que señala si la escucha es un elemento vertebral de la gestión de estas organizaciones y que forma parte de la cultura definida por ellas, se exponen los hallazgos de esta investigación agrupados en diferentes apartados. 


\subsection{Construcción del concepto de escucha organizacional en el retail}

Este subtítulo busca responder y comprender cuál es la noción que se tiene del concepto de escucha a partir de la información obtenida.

Cabe destacar como primer resultado que ningún ejecutivo entrevistado es capaz de definir expresamente desde su perspectiva lo que es la escucha organizacional y tampoco de construir una descripción. Lo que se obtiene al conversar de este tema son conceptos aislados.

Respecto de las ideas más recurrentes que aparecen a partir del análisis, podemos mencionar cuatro:

\subsubsection{Retroalimentación:}

Se vincula a la escucha con la retroalimentación ofeedback que se da a los clientes y colaboradores. Esta tendría que ver con el diálogo entre quienes trabajan en las empresas y la adecuada reciprocidad de las relaciones tal como indican Theunissen \& Noordin (2012).

En primer lugar se obtiene que hay motivación adicional en los colaboradores para trabajar mejor cuando se produce una adecuada retroalimentación (Flynn, Valikoski, \& Grau, 2008).

"Más que generar los espacios y los medios, tiene que ver con una cultura de poder recibir ese feedback y de hacer algo con eso también" (Participante E15, Gerencia de Comunicaciones).

Y al mismo tiempo que esta retroalimentación empuje a mejoras en la realización del trabajo.

"Para nosotros la escucha es feedback, es la capacidad de mostrar oportunidades de mejora tanto en habilidades blandas, como duras" (Participante E3, Gerencia de Marketing).

Esto último ayudaría a los colaboradores a desarrollarse al interior de las compañías entregándoles información de forma permanente respecto de cómo realizan su trabajo. Esto se vería beneficiado cuando existen estructuras organizacionales más horizontales:

"Dentro de nuestra cultura es lo que se está haciendo en las empresas más grandes, que es de puertas abiertas, si alguien de las fuerzas medias del equipo quiere hablar con el gerente de marketing lo puede hacer o con el gerente comercial o de finanzas no hay ningún problema" (Participante E1, Gerencia de Marketing).

En segundo lugar, el concepto de retroalimentación se relaciona con la identificación anticipada de aspectos relevantes para el funcionamiento de la empresa:

"Escuchar para una organización como la nuestra, es ser capaces de interpretar de forma temprana y a partir de toda la información directa o indirecta que pueda estar disponible, lo que la gran mayoría de los clientes realmente quieren y lograr entender para qué lo quieren y cuando lo quieren" (Participante E12, Gerencia de Proyectos Estratégicos).

\subsubsection{Respeto}

La escucha organizacional aparece mencionada como una acción de respeto al interior de las empresas hacia sus colaboradores.

De acuerdo con Gremler y Gwinner (2008) el respeto generaría armonía entre las partes, calidad en las relaciones, comportamientos atentos, de cortesía y buscaría poner aspectos en común para el funcionamiento de la organización.

Desde este punto de vista es percibido por la Dirección de Personas de una de las empresas analizadas que están en el rango secundario de ventas: 
"Creo que la escucha organizacional es parte integral de las organizaciones y empresas ya que, al estar conformadas por personas, la comunicación es inherente a ellas. Las personas quieren ser vistas y escuchadas, y el hacerlo es una señal de respeto" (Participante E2, Gerencia de Recursos Humanos).

Para esta organización es importante darle a cada persona el espacio para que se sienta escuchada y valorada.

\subsubsection{Relación con el cliente}

Entre los conceptos que aparecen visibles a partir del estudio se encuentra que la escucha colaboraría en mejorar el vínculo con el consumidor. La escucha es identificada como una construcción en la empresa para crear una relación colaborativa con el cliente y así dar adecuada forma al servicio que se ofrece (Journée \& Weber, 2017).

Y a partir de esta relación con el consumidor se logra interpretar escenarios y formas de actuar.

"Escuchar para una organización como la nuestra, es ser capaces de interpretar de forma temprana y a partir de toda la información directa o indirecta que pueda estar disponible, lo que la gran mayoría de los clientes realmente quieren y lograr entender para qué lo quieren y cuándo lo quieren" (Participante E16, Gerencia de Comunicaciones).

Esto se entiende en un mercado que como se ha señalado cambia a una velocidad rápida y que es altamente competitivo, por lo que vincularse directamente con el cliente, conocer y entender sus demandas es fundamental. Por lo anterior es importante utilizar herramientas que permitan procesar la información que se recibe. Así los potenciales del Big Data son muy amplios y no solamente enfocados en georreferenciación, "sino también a acceso, caracterización y ponderación con gran precisión de determinados grupos de stakeholders críticos, que afectan y son afectados por los objetivos estratégicos de una empresa" (Portales \& Alcaíno, 2018, p 71).

\subsubsection{Desarrollo de los colaboradores:}

Asimismo, surge la noción a partir del estudio, de que la escucha está ligada al progreso profesional de las personas. Cuando se escucha se puede lograr un adecuado enriquecimiento de las carreras profesionales, al mismo tiempo que al escuchar se logra mejorar las relaciones entre quienes se desempeñan en una misma compañía (Flynn, Valikoski \& Grau 2008).

Su incidencia apunta a efectos sobre la organización:

"Escuchar tiene múltiples consecuencias: desde el punto de vista de la negociación es vital escuchar, ya que sino no entiendo los intereses de la contraparte; desde el punto de vista del equipo sino los escucho no voy a ser un buen líder y yo tengo que sacar el mejor partido a ellos; desde el punto de vista de las áreas de apoyo tienes que darte el tiempo y establecer que las cosas se hagan. Debes conversar con los vendedores en el punto de venta, no puedes saber su estrategia si no los escuchas y es el termómetro fino para saber cómo están funcionando las cosas" (Participante E12, Gerencia de Proyectos Estratégicos).

Al mismo tiempo que colabora en la fidelización de las personas que trabajan, las que perciben que pueden plantear sus inquietudes:

"Para poder lograr que las personas fidelicen de la mejor forma, tienen que sentirse escuchados y tienen que sentir una cosa super humana, sabes qué, decir yo plantee las cosas y puedo, pero no tengo miedo a decir las cosas" (Participante E21, Gerente de Recursos Humanos). 


\subsection{Cultura organizacional que colabora a la implementación del concepto de escucha en el retail}

La cultura de la escucha implica que los ejecutivos de la institución y el departamento de comunicaciones reconozcan la existencia de varios grupos en la organización y de individuos que tienen derecho a hablar y a ser considerados (Macnamara 2015). La cultura es uno de los componentes primarios que orienta una serie de valores, creencias y comportamientos que representan la organización, lo que implica comunicación y en específico escuchar (Welch, \& Mickelson, 2013).

En el caso de las empresas estudiadas sólo dos organizaciones lo manifiestan oficialmente por escrito en sus memorias anuales (Falabella y París), pero no hay precisión respecto de que esta cultura se dé en la práctica y sea algo explícito para quienes trabajan en ella, sino que a través de las entrevistas y cuestionarios sólo se logra obtener apreciaciones generales.

Tal como se mencionó, esta industria se dirige a clientes, colaboradores, proveedores, accionistas, autoridades, comunidad y medios de comunicación. Pero, de las cinco empresas estudiadas clientes y colaboradores se citan por todas ellas como público objetivo. En tanto accionistas, proveedores, comunidad, autoridades y medios de comunicación sólo son mencionadas de manera indistinta en ellas, lo que significa que los énfasis se ponen mayoritariamente en clientes y colaboradores.

A partir de los resultados obtenidos se presentan los siguientes apartados que manifiestan cuáles son los elementos de la cultura que colaborarían a conformar el concepto de escucha en el retail de acuerdo con la información que se obtiene.

\subsubsection{Apertura real a escuchar}

De acuerdo a la información obtenida se advierte un propósito incipiente por parte de las organizaciones y ejecutivos en la búsqueda de la escucha. Sin embargo, se notan distintas percepciones de los ejecutivos sobre cómo lo hace su respectiva organización.

Al consultar sobre la materialización de la cultura de escucha en la empresa se obtienen diferentes respuestas. Por ejemplo para un ejecutivo, la cultura de escucha depende de cada entidad y hay diferencias entre unas y otras:

"El retail se ha pegado un gran salto porque se ha dado cuenta que la información vale oro, que con eso puede presionar cosas, pero hay empresas que lo están haciendo con una mirada en el corto y largo plazo y otras solamente lo están haciendo a nivel de subsistencia. Hay un período de transición donde algunos alumnos van más aventajados que otros" (Participante E4, Gerencia de Marketing).

En el caso de un ejecutivo que trabaja en la omnicanalidad no todos los actores internos tienen la misma apertura o interés por realizarla:

"Yo creo que hemos ido mutando, en el retail todos queremos escuchar al cliente, pero no sé si todas las capas lo tienen tan claro. No sé si el asesor que está de cara al cliente en la tienda le queda claro que lo tenemos que escuchar para saber cómo lo servimos mejor" (Participante E 22, Gerencia de Omnicanalidad).

Asimismo, también se considera como un proceso que debe ejecutarse de forma contínua:

"Es un tema que le hemos dado varias vueltas. Yo creo que hoy, con los tiempos que estamos viviendo, con toda la tecnología que hay y que este cliente omnicanal tiene mucha facilidad de información es clave tomar decisiones preguntándole a los clientes o por lo menos tomando la información que entregan" (Participante E13, Gerencia de Marketing).

Con respecto a la real apertura a escuchar el gráfico 1 refleja la percepción que tienen los ejecutivos entrevistados de la cultura de la empresa expresada en cuatro categorías que van desde el monó- 
logo hasta el full diálogo. Al respecto, cabe recordar como señala Burnside-Lawry (2012) que el diálogo lleva implícito escucha activa e intercambio de conocimiento.

Gráfico 1. Cultura de la empresa apunta a monólogo o diálogo

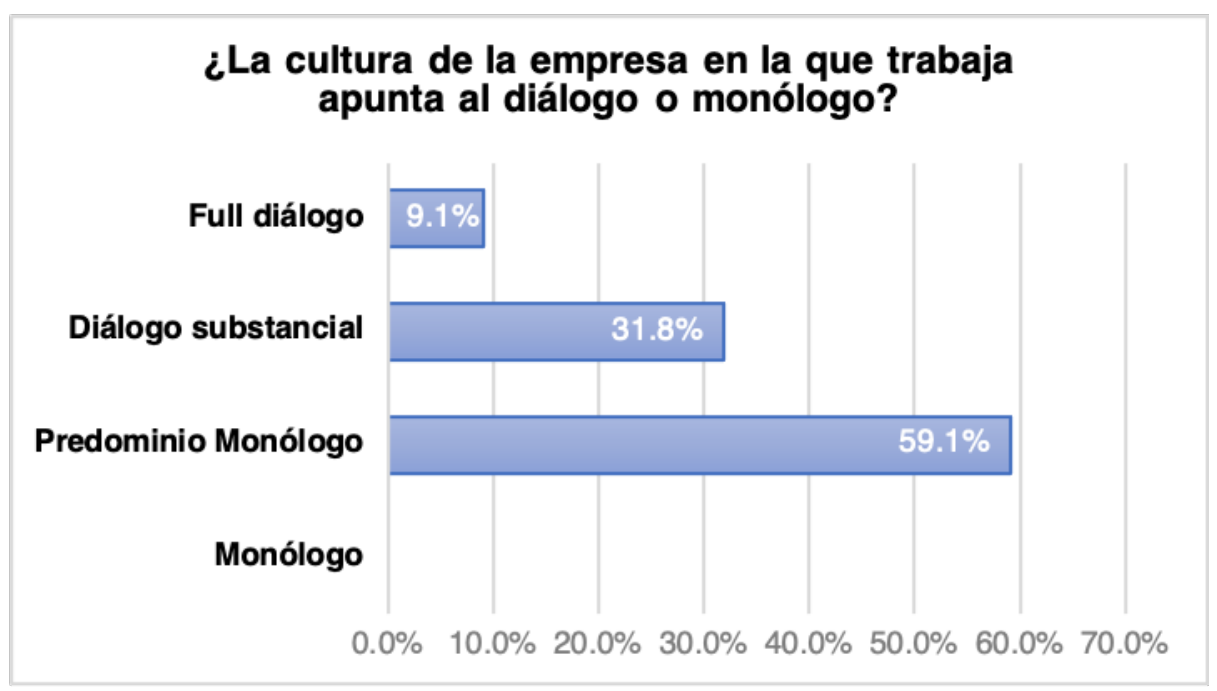

Fuente: Elaboración propia a partir de la información de los cuestionarios

Observamos que los ejecutivos declaran una presencia mayoritaria de predominio del monólogo en las compañías. Esto estaría generado por culturas organizacionales que no tienen el concepto de escucha tan interiorizado como un elemento del management o porque no hay esa apertura para detenerse a escuchar de forma adecuada.

Los ejecutivos creen que parte de la causa de este predominio del monólogo se debe a tiempo y recursos escasos, frente a una exigencia importante de resultados.

\subsubsection{Entender qué significa escuchar y una cultura para ello en la organización}

Como se ha indicado existe , a partir de los datos obtenidos, una percepción de que se necesita escuchar, pero los conceptos asociados a ella son variados (ver 4.1)

Como indica Gillchrist y Van Hoeven (1994) la escucha puede entenderse como una característica relacionada sólo a determinadas habilidades de liderazgo de algunos trabajadores, pero no ser algo intrínseco a las singularidades y valores fundamentales de la empresa.

Es posible constatar diversidad de opiniones acerca de ella hasta en una misma compañía lo que refleja una línea poco clara de la definición y incorporación de ella desde el gobierno corporativo. Para ejemplificar lo anterior, observamos dos colaboradores que tienen visiones diferentes respecto a la cultura de la escucha que se ejecuta en una misma empresa.

"En esta organización si se quiere ir hacia una cultura de escucha, nuestro claim es el cliente al centro de todo, y si se hacen cosas para poder escuchar al cliente. Y en el caso de los colaboradores, es difícil porque el back office y la tienda funcionan de forma diferente, son de naturaleza diferente". (Participante, E14, Gerencia Omnicanal).

En contraposición a lo anterior, en la misma entidad:

"Yo venía de la industria de malls, donde el cliente estaba al centro. Cuando llegué a esta compañía recién se puso el cliente al centro, lo que me pareció tardío porque yo venía de una empresa, donde eso ocurría hace años" (Participante E3, Gerencia de Marketing). 
Si bien las dos partes creen que el cliente está al centro, uno sostiene que la compañía ha avanzado, mientras que el otro a cargo de marketing ve que la decisión de poner al cliente al centro es tardía respecto de otras compañías del rubro que ya lo están haciendo.

Otra línea que se verifica indica que no habría cultura de escucha debido a que estas empresas de retail forman parte de grandes conglomerados que a su vez tienen otras unidades de negocio - financiero, seguros, mejoramiento del hogar, inmobiliario- por lo que es muy difícil establecer una política general para todos dadas las características específicas de cada uno. Esta premisa dificulta el concepto planteado por Gillchrist \& Van Hoeven, (2012) que apunta a la escucha como un valor al interior de la organización que se decide, comanda por alguien y aunque sea complejo trata de medirse.

Este ejecutivo, que proviene de una empresa que declara oficialmente una cultura por escuchar, cree que al ser una compañía corporativa, el concepto se diluye.

"Esta empresa tiene como dicotomía que no sabe si es un holding o una corporación. Para mí es una corporación. Nuestra compañía tiene una cultura de puertas abiertas de tratar de escuchar a la gente, pero eso no se repite necesariamente en todas las partes del grupo, hay áreas que no tienen esa cultura y se entra en la lógica de los caballazos para mover las cosas" (Participante E7, Gerencia de E-Commerce).

Desde las entrevistas se advierte una confusión entre comunicar y escuchar. La escucha forma parte de la comunicación y debe entenderse como una comprensión simbólica e interpretativa de ella y no meramente como algo relacional o instrumental (Borner \& Zerfass, 2018). En este sentido llama la atención que una Dirección de Comunicaciones - que debiera ser el órgano que mejor entiende la necesidad de escuchar y que genera más ventas-, declare que más que escuchar, el énfasis está puesto en informar:

"No hay tanta cultura de escucha, hay un desafío importante. En general nosotros informamos, nos preocupamos de hacer diagnósticos, pero no hay ningún mecanismo formal para escuchar". (Participante E18, Gerencia de Comunicaciones).

\subsubsection{Satisfacción de los requerimientos que plantean los clientes}

Con los consumidores, la escucha apunta a una satisfacción de las necesidades subyacentes y la construcción de relaciones entre la empresa y el consumidor (Barbour, 2017).

Esto es posible de evidenciar en todas las compañías estudiadas, lo que se refleja en las siguientes citas de sus documentos públicos. París , los considera como algo relevante, en el plan estratégico:

"Consideramos a los clientes como nuestra inspiración y razón de ser. Para nosotros, entregarles la mejor experiencia es hacer que se sientan sorprendidos, valiosos y diferentes. Nos seduce escucharlos constantemente y entender sus anhelos y tendencias, porque queremos ser los primeros en satisfacerlos (Reporte Conciencia Celeste 2017, p.35).

Al mismo tiempo que La Polar se centra en dos grupos específicamente entre los que se encuentran los clientes:

"La Polar debe ser valorada y respetada por sus grupos de interés, partiendo por los colaboradores y clientes. Su satisfacción es el centro del negocio y debe trabajar de manera responsable para cumplir con sus expectativas y servirlos cada día mejor (La Polar, Memoria anual, 2017, p. 13).

La eficacia en la construcción de una cultura de escucha del consumidor está estrechamente relacionada e impulsada por el mercado en el que se desempeña la empresa y la orientación al consumidor (Randall, 2020). 
En este sentido es posible visualizar que es importante para estas compañías tener respuestas dinámicas con los clientes :

"Procuramos, además tener canales de comunicación directos, ágiles y expeditos con nuestros clientes, para que exista una retroalimentación continua y eficiencia en la respuesta a sus requerimientos" (Falabella, Memoria 2017, p.3).

\subsubsection{Acciones que colaboran a una escucha efectiva}

Para realizar una escucha activa es importante como indica Macnamara (2016) contar con recursos, tecnología y procesos que colaboren a la ejecución de ella.

A partir de los cuestionarios tomados como muestra se recopilan las acciones que estas cinco instituciones realizan de forma más recurrente, al mismo tiempo de cómo trabajan y con cuál periodicidad estos instrumentos.

La tabla 1 recoge las acciones que se realizan con mayor frecuencia. Las entrevistas y en especial los cuestionarios permiten determinar que los instrumentos más utilizados son la página web de las compañías y las redes sociales.

Tabla 1. Acciones realizadas por las empresas en relación a la escucha organizacional

\begin{tabular}{|l|l|}
\hline Página Web & - En promedio un 45,5\% de las compañías responde solicitudes con periodicidad diaria \\
& o semanal. \\
& - $50 \%$ de las compañías no contesta nunca las preguntas que reciben. \\
\hline Redes Sociales & - Instagram: se utiliza en las cinco empresas para presentar productos. \\
& - Facebook: un 54,5\% de las empresas da respuesta diaria o semanal; $40,9 \%$ no con- \\
& testa. \\
& - Twitter: $50 \%$ de las empresas da respuesta diaria o semanal; $50 \%$ no responde. \\
\hline Otros instrumentos & - Información verbal de lo que ocurre en la tienda entre trabajador y supervisor: diaria. \\
& - Whatsapp: comunicación tienda y jefatura: diaria. \\
& - Instagram: para colaboradores: diaria. \\
- Encuestas tomadas a clientes en todas las empresas: mensuales y/o trimestrales \\
dependiendo de la organización. \\
- Uso de Inteligencia artificial: esporádico, depende de la organización.
\end{tabular}

Fuente: Elaboración propia a partir de los cuestionarios

Con respecto al tiempo dedicado a escuchar y que nos permite determinar si se está construyendo una cultura de escucha en las empresas, solo un tercio de los entrevistados manifiesta que el tiempo total de la jornada que se dedica a escuchar y responder a sus equipos de trabajo supera el $50 \%$ del total.

\section{Discusión y Conclusiones}

La escucha organizacional como instrumento que construye cultura organizacional tiene consecuencias para las instituciones.

Es posible advertir que para los ejecutivos entrevistados hay conciencia que la comunicación debe ser bidireccional y retroalimentada permanentemente y que en el caso de la industria del retail, la tendencia general apunta a tratar de operar de esa forma. Pero lograr una comunicación bidireccional no siempre es fácil y depende del tipo de público. De las entrevistas se desprende que las gerencias de comunicaciones tienen más claro la importancia de la bidireccionalidad para el funcionamiento y sustentabilidad de las compañías. 
Con respecto al objetivo de esta investigación sobre la percepción de los ejecutivos de las empresas estudiadas y la presencia de una cultura de la escucha en las organizaciones en las que trabajan. Se aprecia que los ejecutivos entienden la importancia de escuchar, que es un concepto que se ha ido instalando en las compañías, pero que aún falta para lograrlo y sistematizarlo.

La razón se debe principalmente a que no existen estructuras definidas para escuchar, sino herramientas que permiten obtener información. Tampoco se destinan suficientes recursos como son tiempo, dinero, equipos, canales, capacitación etc. Sin embargo, eso debiera tender a cambiar, ya que por ejemplo consumidores y empleados hoy se expresan más y tienen mayor cantidad de formas de hacerlo.

En este sentido las organizaciones deben buscar mayor apertura para una escucha activa, tratar de colocar el concepto de escucha bajo una misma perspectiva para todos quiénes integran la organización de modo de uniformar criterios, entender qué significa satisfacer la necesidad del cliente y finalmente consolidar mayor cantidad de acciones que colaboren con la escucha.

Las entrevistas muestran que el concepto de escucha que se maneja significa más una necesidad de la empresa, que un aporte para el mejor funcionamiento de ellas. La exigencia por escuchar - está dada por las condiciones de alta competencia en que se desarrolla este negocio- y no necesariamente es una prioridad de las compañías. Coinciden en esta mirada tanto el área de desarrollo de proyectos, como marketing que ven la escucha como una necesidad:

"Esta es una empresa que escucha a todos, pero no necesariamente porque quiera o por convicción, sino porque lo necesita, porque no hay otra forma de competir". (Participante E16, Gerencia de Comunicaciones).

En relación a la pregunta de investigación que busca conocer si las organizaciones de retail chileno consideran la escucha como un elemento vertebral de su gestión y que forma parte constitutiva de la cultura organizacional definida por ellas. Cabe consignar que el concepto de escucha ha permeado en la industria del retail como un intangible importante que tiene que estar presente. Existe conciencia respecto a la relevancia de escuchar, pero en el caso de las empresas analizadas no se pone a la escucha en un lugar relevante de la gestión y administración. Así lo demuestra que de las interacciones entre ejecutivos y empleados más del $60 \%$ corresponde a predominio del monólogo y entrega de información. Las razones esgrimidas apuntan a la alta competencia, márgenes de utilidad apretados y un modelo de venta que cambia de forma muy rápida en la industria y que exige adaptación.

Ante esto, es importante lograr una conexión entre las estrategias de comunicación y el devenir diario de la organización. Como señalan Falkheimer \& Heide (2018), la comunicación es un requisito para la existencia de la organización, puede ser formal e informal entre los miembros de la institución y sus públicos, las conversaciones y los textos producen y reproducen a la organización, cuando los miembros de una institución se comunican genera en ellos y otros un dibujo acerca de lo que es la institución, su estructura, su clima, cómo son percibidos por la competencia, sus cualidades, sus procesos y su futuro. Se debe procurar avanzar más allá de que las teorías de comunicación corporativa y relaciones públicas que enfatizan la relevancia de la interacción bidireccional, las relaciones, el compromiso, el diálogo y la reciprocidad, y dejar de lado los modelos de evaluación en el campo que se enfocan estrechamente en las metas y objetivos de la organización (Macnamara, 2020).

\section{Limitaciones y futuros estudios}

Estudiar y comprender cómo se está realizando la escucha en el sector retail se consignó como una tarea difícil de abarcar para una investigación de esta envergadura. Por lo mismo se limitó el estudio de ciertos grupos de interés para dejar el análisis del resto de los públicos para futuras investigaciones. 
La industria del retail en Chile resguarda su información de forma estricta, por lo que el acceso a datos de cada institución es restringido y no es de carácter público. Esto significó ajustes durante el inicio del trabajo de campo y respetar estrictamente la confidencialidad exigida por los participantes a la hora de publicar los resultados.

Sería interesante conocer qué ocurre en el resto de los países latinoamericanos y si su idiosincrasia local afecta positiva o negativamente a la cultura de la escucha en este tipo de organizaciones.

Resulta estimulante para el futuro continuar estudiando otras industrias de consumo y cuál es la relevancia de desarrollar procesos de escucha en ellas. En el caso de esta investigación sobre el retail, por sus características requiere y debiera demandar la consolidación de culturas y políticas de escucha al interior de las compañías de modo de entender los cambios de hábito de consumo que se generan permanentemente y que evolucionan de forma rápida. En contraposición a esto puede haber otros mercados para los cuáles este intangible sea menos relevante de implementar por cuanto se requiere de menos información de los diferentes públicos para su funcionamiento diario.

Finalmente, el proceso de comunicación en las instituciones se consolida como parte de los procesos estratégicos de las organizaciones, por lo que hay un desafío importante tanto para el management de las empresas, como para quiénes se encargan de las comunicaciones de las mismas.

\section{Bibliografía}

Apolo, D., Báez, V., Pauker, L., \& Pasquel, G. (2017). Gestión de Comunicación Corporativa: consideraciones para el abordaje de su estudio y práctica. Revista Latina de Comunicación Social, (72), 521-539.

Banco Mundial. (2020). Comercio de Mercaderías (\% del PIB). https://datos.bancomundial.org/ indicador/TG.VAL.TOTL.GD.ZS

Barbour, J. B. (2017). Listening and Organizing. The International Encyclopedia of Organizational Communication, 1-5. https://doi.org/10.1002/9781118955567.wbieoc126

Borner, M \& Zerfass, A. (2018). The Power of Listening in Corporate Communications: Theoretical Foundations of Corporate Listening as a Strategic Mode of Communication. Public Relations and the Power of Creativity (Advances in Public Relations and Communication Management. Vol 3). Emerald Publishing Limited. 3-22. https://doi.org/10.1108/S2398$\underline{391420180000003001}$

Botan, C.H., \& Taylor, M. (2004). Publics relations: State of the field. Journal of Communication, 54(4), 645-661. https//doi.org/ 10.1111/j.1460-2466.2004.tb02649.x

Brownell, J. (2008). Exploring the strategic ground for listening and organizational effectiveness. Scandinavian Journal of Hospitality and Tourism, 8(3), 211-229. https://doi.org/10.1080/ 15022250802305295

Bryman, A. (2012). Social Research Methods. Oxford University Press.

Burgos Romero, C. (2018). La Responsabilidad Social o sostenibilidad: un enfoque desde el entorno y la comunicación. RETOS. Revista de Ciencias de la Administración y Economía, 8(16), 47-59.

Burnside-Lawry, J. (2012). Listening and participatory communication: A model to assess organization listening competency. International Journal of Listening, 26(2), 102-121. https://doi. org/10.1080/10904018.2012.678092 
Bussey, N. (2010). Dialogue as a basis for stakeholder engagement: Defining and measuring the core competencies. In R.L Heath (Ed.), The SAGE Handbook of Public relations 127-144, Sage.

Claro, C. (2019). La escucha organizacional: una propuesta conceptual. Anagramas Rumbos y Sentidos de la Comunicación, 17(34), 239-253. https://doi.org/10.22395/angr.v17n34a12

Corbetta, P. (2007). Metodología y Técnicas de Investigación Social. Mc Graw Hill.

Denzin, N. K., \& Lincoln, Y. S. (1994) Introduction: Entering the Field of Qualitative Research en Denzin, N. K., Lincoln (eds.) Handbook of Qualitative Research. California: Sage.

Falkheimer, J., \& Heide, M. (2018). Strategic Communication: an introduction. Routledge.

Flynn, J., Valikoski, T. R., \& Grau, J. (2008). Listening in the business context: Reviewing the state of research. The International. Journal of Listening, 22(2), 141-151. https://doi. org/10.1080/10904010802174800

Gilchrist, J. A., \& Van Hoeven, S. A. (2012). Listening as an organizational construct. International Listening Association. Journal, 8(1), 6-30. https://doi.org/10.1080/10904018.1994.10499129

Gremler, D. D., \& Gwinner, K. P. (2008). Rapport-building behaviors used by retail employees. Journal of Retailing, 84(3), 308-324. https://doi.org/10.1016/j.jretai.2008.07.001

Gutiérrez-García E; Recalde, M. \&, \& Piñera-Camacho, A. (2015). Reinventing the wheel? A comparative overview of the concept of dialogue. Public Relations review, 41, 744-753.

Gutiérrez-García, E. (2010). Dimensión comunicativa del buen gobierno empresarial. Revista Empresa y Humanismo, XIII(2/10), 149-182. https://doi.org/10.1016/j.pubrev.2015.06.006

Grunig, J., \& Hunt, T. (1984). Managing public relations. Harcourt Brace Jovanovich College Publishers.

Heath, R. L., Pearce, W. B., Shotter, J., Taylor, J. R., Kersten, A., Zorn, T., Deetz, S. (2006). The process of dialogue. Management Communication Quartely. 19(3), 341-375. https://doi. org $/ 10.1177 / 0893318905282208$

Hernández Sampieri, R., Fernández Collado, C., \& Baptista Lucio, P. (2010). Metodología de la investigación. Mc Graw Hill.

Hoyt, J., \& Gerloff, E. A. (1999). Organizational environment, changing economic conditions, and the effective supervision of technical personnel: A management challenge. The Journal of High Technology Management Research, 10(2), 275-293. https://doi.org/10.1016/S1047-8310(99)00014-0

Hunt, G. T., \& Cusella, L. P. (1983). A field study of listening needs in organizations.

Communication Education, 32(4), 393-401. https://doi.org/10.1080/03634528309378560

Itani, O. S., Goad, E. A., \& Jaramillo, F. (2019). Building customer relationships while achieving sales performance results: Is listening the holy grail of sales? Journal of Business Research, 102, 120-130.

Janakiraman, N., Bullemore, J., Valenzuela-Fernández, L., \& Jaramillo, J. F. (2019). Listening and perseverance-two sides to a coin in quality evaluations. Journal of Consumer Marketing.

Johnston, M. K., Reed, K., \& Lawrence, K. (2011). Team listening environment (TLE) scale: Development and validation. Journal of Business Communication, 48, 3-26. https://doi. org/10.1177/0021943610385655 
Journée, R., \& Weber, M. (2017). Co-creation of experiences in retail: Opportunity to innovate in retail business. In Managing Complexity, 391-404, Springer, Cham.

Macnamara, J. (2020). Embracing evaluation theory to overcome "stasis": Informing standards, impact and methodology. Corporate Communications: An International Journal, 25(2), 339-354.

Macnamara, J. (2019). Explicating listening in organization-public communication: Theory, practices, technologies. International Journal of Communication.

Macnamara, J. (2016). Organizational listening. The Missing Essential in Public Communication. Peter Lang Publishing.

Macnamara, J. (2015). Creating an 'architecture of listening' in organizations: The basis of engagement, trust, healthy democracy, social equity, and business sustainability. Sydney, NSW, University of Technology.

Macnamara, J. (2014). Organizational listening: A vital missing element in public communication and the public sphere. Asia Pacific Public Relations Journal, 15(1), 89-108.

Moreno, Á., Khalil, N., \& Gómez, M. D. P. (2018). La escucha organizacional como estrategia de comunicación. Análisis de su gestión en España/The organizational listening as a communication strategy. Analysis of its management in Spain. Revista internacional de relaciones públicas, $8(15)$, 197-214.

Palmatiere, R., Rajiv P.D, Dhruv G \& Kenneth E. (2006). Factors Influencing the Effectiveness of Relationship Marketing: A Meta-Analysis. Journal of Marketing. 70 (October), 136-153.

Palmatiere, R. (2008). Relationship Marketing. Cambridge, Massachusetts: Marketing Science Institute.

Portales, J. C., \& Alcaíno, J. C. (2018). El rol del Big data como herramienta de comunicación estratégica y construcción de reputación en Chile. Revista ComHumanitas, 9(2), 66-84.

Purdy, M. W., \& Manning, L. M. (2015). Listening in the multicultural workplace: A dialogue of theory and practice. International Journal of Listening, 29(1), 1-11. https://doi.org/10.1080/10904 $\underline{018.2014 .942492}$

Randall, D. (2020) The Current State of Corporate Voice of the Consumer Programs: A Study of Organizational Listening Practices and Effectiveness, International Journal of Listening,34(3), 156-177. http:// 10.1080/10904018.2018.1482747

Rojas, G. (2017). Entre la conversación y el diálogo: algunos aspectos para la escucha. Enunciación, 22(2), 189-201.

Romero-Rodríguez, L. M., \& Castillo-Abdul, B. (2019). Comunicación para la motivación. Claves de la asertividad y del trabajo en equipo en las organizaciones. R. Ravina-Ripoll, LB Tobar Pesántez y E. Nuñez Barriopedro (Coords.), Happiness Management and Creativity in the XXI Century. Intangible capitals as a source of innovation, competitiveness and sustainable development, 41-54.

Schein, E. (2010). Organizational culture and leadership (4rd ed.). Jossey-Bass.

Schein, E. (1993). On dialogue, culture, and organizational learning. Organizational dynamics, 22(2), 40-51. https://doi.org/10.1016/0090-2616(93)90052-3

Schein, E. (1992). Organizational culture and leadership. Jossey-Bass. 
Strandberg, L. (2010). El compromiso con los grupos de interés. Cuadernos de la Cátedra "la Caixa” de Responsabilidad Social de la Empresa y Gobierno Corporativo (Nº10), 14-17.

Susanne, T. \& Ellerup, A. (2011). Strategic stakeholder dialogues: a discursive perspective on relationship building. Corporate Communications: An International Journal, Vol. 16 No. 3, 204217. https://doi.org/10.1108/13563281111156871

Taylor M \& Kent, M. (2014). Dialogic Engagement: Clarifying Foundational Concepts. Journal of Public Relations Research, 26(5), 384-398. https://doi.org/10.1080/1062726X.2014.956106

Theunissen, P. \&, \& Noordin, W. (2012). Revisiting the concept "dialogue" in public relations. Public Relations review, 38(1), 5-13. https://doi.org/10.1016/j.pubrev.2011.09.006

Trenholm, S., \& Jensen, A. (2008). Interpersonal communication. Oxford University Press.

Villagra N; López B \& Monfort A. (2015). La gestión de intangibles y la marca corporativa: ¿ha cambiado algo en la relación entre las empresas y la sociedad? Revista Latina de Comunicación Social, 70, 793-812. https://doi.org/ 10.4185/RLCS-2015-1072

Welch, S. A., \& Mickelson, W. T. (2013). A listening competence comparison of working professionals. International Journal of Listening, 27(2), 85-99. https://doi.org/10.1080/10904018.2 $\underline{013.783344}$

Worthington, D., \& Fitch-Hauser, M. (2018). Listening Processes, Functions, and Competency. Routledge.

Yang, S \& Kang, M. (2015). A Study on Dialogic Communication, Trust, and Distrust: Testing a Scale for Measuring Organization-Public Dialogic Communication (OPDC). Journal of Public Relations Research, 27, 175-192. https://doi.org/10.1080/1062726X.2015.1007998 